Highlights of Astronomy, Vol. 13

International Astronomical Union, 2003

O. Engvold, ed.

\title{
An Accurate Model of Mercury's Spin-Orbit Motion
}

\author{
Nicolas Rambaux \& Eric Bois \\ Observatoire Aquitain des Sciences de l'Univers, UMR L3AB \\ CNRS/INSU 5804, B.P. 89, F-33270 Floirac France
}

\begin{abstract}
Our work deals with the physical and dynamical causes that induce librations of Mercury around an equilibrium state defined by the 3:2 spin-orbit resonance. In order to integrate the spin-orbit motion of Mercury, we have used our gravitational model of the solar system including the Moon's spin-orbit motion. This model, called SONYR (acronym of Spin-Orbit N-bodY Relativistic model), was previously built by Bois, Journet and Vokrouhlickỳ in accordance with the requirements of the Lunar Laser Ranging observational accuracy.

Using the model, we have identified the main perturbations acting on the spin-orbit motion of Mercury such as the planetary interactions or the dynamical figure of the planet. Moreover, the complete rotation of Mercury exhibits two proper frequencies, namely 15.847 and 1066 years, and in addition one spin-orbit secular resonance (298 898 years). A new determination of the mean obliquity of Mercury has been proposed. Besides, we have identified in the Hermean librations the impact of the uncertainty of the greatest principal moment of inertia $\left(\mathrm{C} / \mathrm{MR}^{2}\right)$ on the obliquity as well as on the libration in longitude (2.3 mas and 0.45 as respectively for an increase of $1 \%$ on the $\mathrm{C} / \mathrm{MR}^{2}$ value). These accurate relations have to be taken into account in the context of the two upcoming missions BepiColombo and MESSENGER.
\end{abstract}

\section{Introduction}

The 3:2 spin-orbit resonance between the rotational and orbital motions of Mercury results from a functional dependence of the tidal friction adding to a non-zero eccentricity and a permanent asymmetry in the equatorial plane of the planet (Balogh and Giamperi, 2002). The upcoming space missions, MESSENGER (Solomon et al., 2001) and BepiColombo (Milani et al., 2001) with on-board instrumentation capable of measuring the Hermean rotational parameters stimulate the objective to reach an accurate theory of the rotational motion of Mercury. We use our BJV relativistic model of solar system integration (first post-Newtonian approximation level) including the spin-orbit coupled motion of the Moon (Bois and Vokrouhlickỳ, 1995; Bois, 2000). We extended this model to the spin-orbit couplings of the terrestrial planets Mercury, Venus, the Earth, the Moon and Mars; this updated model is at present called SONYR (acronym of Spin-Orbit N-BodY Relativistic model). The SONYR model permits to identify the different families of rotational librations in the spin-orbit motion of Mercury by clearly analyzing their causes (Rambaux and Bois, 2003). 

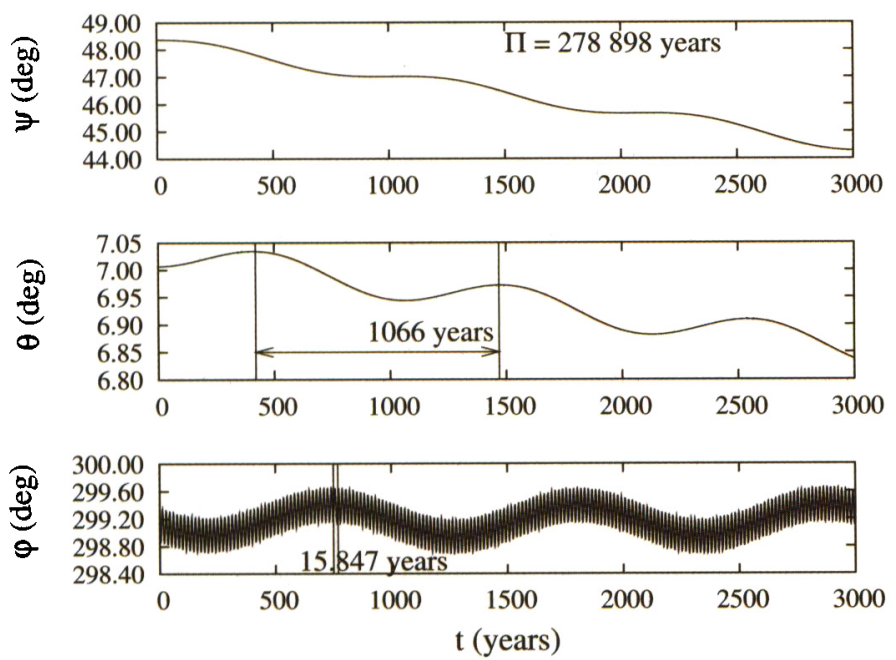

Figure 1. The rotational motion of Mercury in the 3-1-3 Eulerian sequence $(\psi, \theta, \varphi)$. The reference frame is given by the ecliptic $\mathbf{J} 2000$.

\section{The Mercury's spin-orbit motion and its instantaneous obliquity}

The figure 1 presents the rotational motion of Mercury in the usual 3-1-3 Eulerian sequence $(\psi, \theta, \varphi)$. The spin-orbit resonance of Mercury is characterized by two proper frequencies, namely 15.847 years and 1066 years. Moreover, the dynamical behavior of Mercury presents a secular variation of 278898 years, which is due to the nodal precession of the equatorial plane of Mercury relative to the ecliptic plane. This secular variation can be understood as a spin-orbit secular resonance playing the role of a second synchronism.

In integrating the SONYR model, we also obtain the dynamical behavior of the $\eta$ Hermean instantaneous obliquity. The dynamical evolution of $\eta$ is presented in Figure 2. The dynamical behavior of $\eta$ makes in evidence a sine function according to a long period of 1066 years with an amplitude of 0.5 amin. The resulting mean obliquity $\eta_{\text {mean }}$ of Mercury is 1.665 arc-minutes. Let us emphasize that the large amplitude of $\eta$ could seriously make ambiguous the determination of $\eta_{\text {mean }}$ by space missions.

\section{The librations of Mercury}

We have identified in the Hermean librations the impact of the variations of the greatest principal moment of inertia $\left(\mathrm{C} / \mathrm{MR}^{2}\right)$ on the obliquity as well as on the libration in longitude $\varphi$, as follows :

$$
\Delta\left(\mathrm{C} / \mathrm{MR}^{2}\right)=1 \% \Longrightarrow \begin{array}{ll}
2.3 \operatorname{mas} \text { on } \eta \\
0.45 \text { as } & \text { on } \varphi
\end{array}
$$




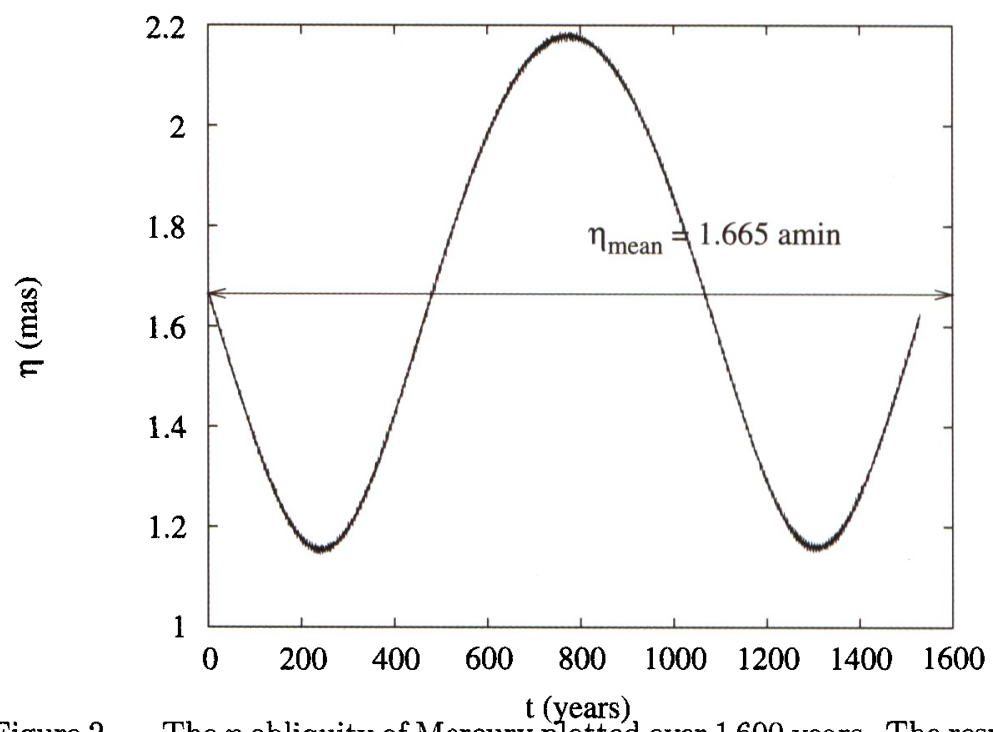

Figure 2. The $\eta$ obliquity of Mercury plotted over 1600 years. The resulting mean obliquity of Mercury is 1.665 arc-minutes.

Such librations belong to the family of potential librations according to the general terminology given in Bois (1995). One of the main objectives of the BepiColombo and MESSENGER missions is to measure the rotation parameters of Mercury up to an accuracy allowing to constrain size and physical state of the Hermean core (Solomon et al., 2001; Milani et al., 2001). In order to reach such an objective, the BepiColombo mission has to obtain a value of the $\mathrm{C} / \mathrm{MR}^{2}$ coefficient with an accuracy of 0.003 , i.e. $1 \%$. As a consequence, this mission foresees measuring the libration angle and the obliquity with an accuracy of 3.2 and 3.7 arcsec respectively (Milani et al., 2001). According to (1) coming from SONYR, these two signatures are very faint and probably too much with respect to the accuracy expected by the space missions.

\section{References}

Balogh, A., \& Giamperi, G., 2002, Rep.Prog.Phys., 65, 529

Bois, E., \& Vokrouhlicky, D., 1995, A \& A, 300, 559

Bois, E., 1995, A \& A 296, 850

Bois, E., 2000, C. R. Acad. Sci. Paris, T. 1, Serie IV, p. 809-823

Milani, A., Vokrouhlicky, D., \& Bonanno, C., 2001, Planetary and Space Science, 49, 1579

Rambaux, N., \& Bois, E., A \& A, 401, 381

Solomon, S.C., et al., 2001, Planetary and Space Science, 49, 1445 